
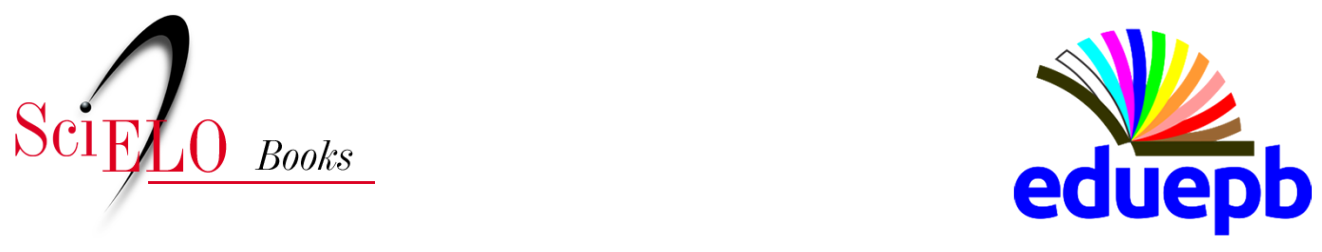

\title{
Do Brasil para o Mundo \\ Osvaldo Sunkel - Um intelectual indispensável para entender o mundo das últimas décadas
}

Jean De Mulder Fuentes

\section{SciELO Books / SciELO Livros / SciELO Libros}

FUENTES, J. M. Osvaldo Sunkel - Um intelectual indispensável para entender o mundo das últimas décadas. Interviewed: Osvaldo Sunkel. In: SOUSA, C. M., THEIS, I. M., and BARBOSA, J. L. A., eds. Celso Furtado: a esperança militante (Depoimentos): vol. 2 [online]. Campina Grande: EDUEPB, 2020, pp. 285-295. Projeto editorial 100 anos de Celso Furtado collection. ISBN: 978-65-86221-11-4. https://doi.org/10.7476/9786586221671.0013.

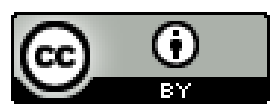

All the contents of this work, except where otherwise noted, is licensed under a Creative Commons Attribution 4.0 International license.

Todo o conteúdo deste trabalho, exceto quando houver ressalva, é publicado sob a licença Creative Commons Atribição 4.0.

Todo el contenido de esta obra, excepto donde se indique lo contrario, está bajo licencia de la licencia Creative Commons Reconocimento 4.0. 


\section{Osvaldo Sunkel ${ }^{\star}$}
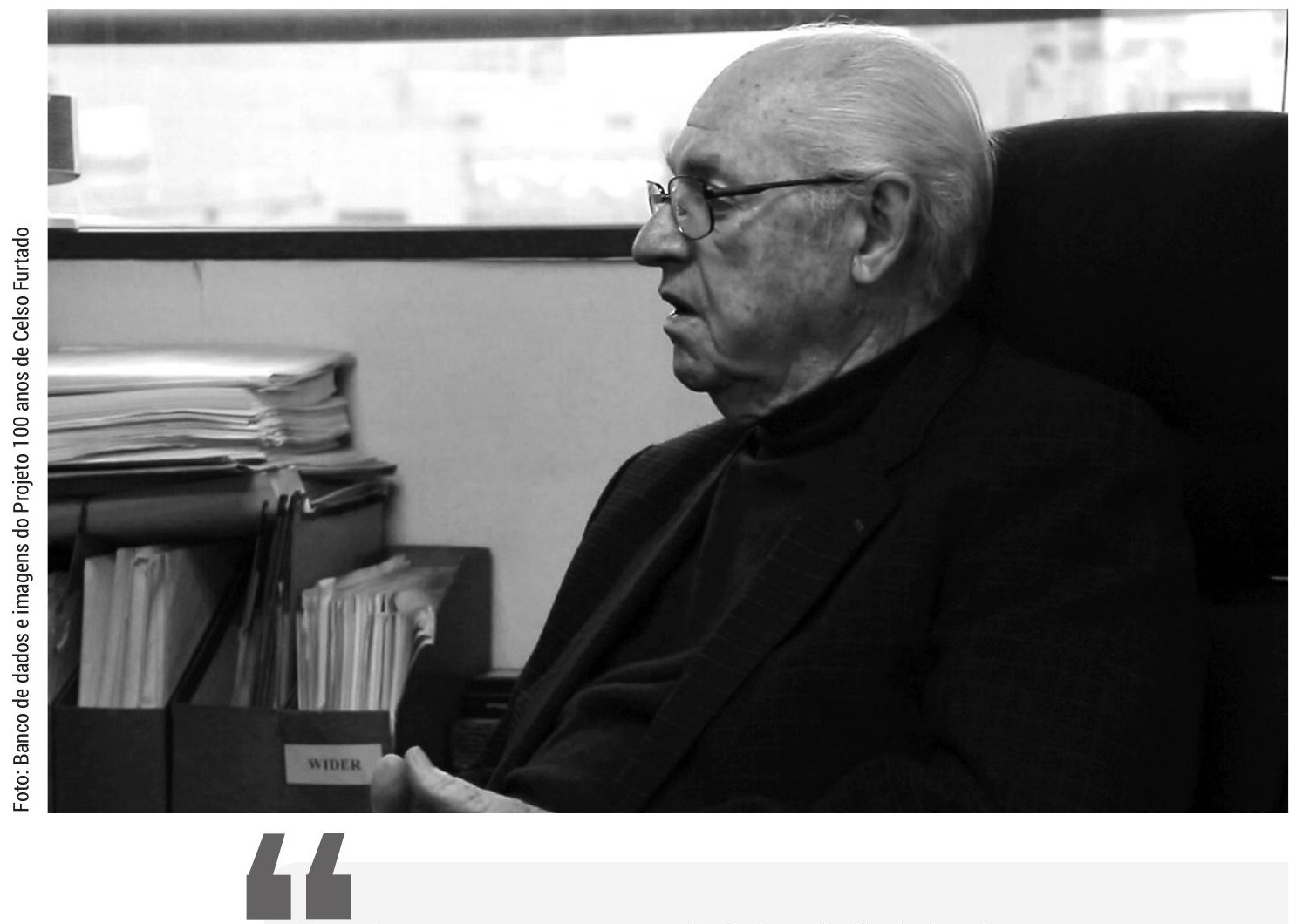

Eu acho que o pensamento de Celso ainda é absolutamente válido. É uma leitura obrigatória para qualquer pessoa interessada em desenvolvimento hoje. Eu certamente gostaria de perguntá-lo sobre tudo isso que vem acontecendo nos últimos 20 anos, que está reconfigurando completamente o mundo, com grandes transformações geoeconômicas, políticas e demográficas... Celso insistiu muito na possibilidade de que os países da AméricaLatina tenham um pensamento e uma cultura de desenvolvimento que vêm de dentro.

* Entrevista realizada em 10/o6/2014, na sede da Cepal em Santiago, no Chile, por Jean Henri Benoît de Mulder Fuentes, no contexto das atividades do Procondel (Sudene UFPE) e cedida aos organizadores da Trilogia: Celso Furtado: a esperança militante. 


\section{Um intelectual indispensável para entender o mundo das últimas décadas}

Jean De Mulder Fuentes ${ }^{1}$

$\mathrm{O}$ svaldo Sunkel nasceu em Puerto Montt, Chile, em 1929. Estudou Economia e Administração na Universidade do Chile e fez pósgraduação na ECLAC e na London School of Economics and Political Science. Desde 1952 está ligado à CEPAL e à Universidade do Chile, entre 1975 e 1986 ao Instituto de Estudos de Desenvolvimento da Universidade de Sussex, e desde 1987 à Development Research Corporation (CINDE), da qual é presidente desde 1991. Para a ECLAC trabalhou em muitos países da América Latina, especialmente no México, Brasil, Chile, Panamá e América Central. Foi fundador e Diretor do Escritório da Cepal no Brasil entre 1959 e 1961, e ingressou, desde sua criação, em 1962, no Instituto Latino-americano de Planejamento Econômico e Social (ILPES), no qual dirigiu o Programa de Formação e, posteriormente, o Projeto de Pesquisa em Desenvolvimento; ele então criou e liderou a Unidade de Desenvolvimento e Meio Ambiente do ECLAC/ PNUMA (1978-1987).

Em 1987 foi nomeado pela ECLAC e pelo Ibero-American Cooperation Institute Director of Ibero-American Thought - Journal of Political Economy e de 1988 a 2002 atuou como Conselheiro Especial do Secretário Executivo da CEPAL. Na Universidade do Chile iniciou sua atividade acadêmica em 1955 na Faculdade de Ciências Econômicas e Administrativas tornando-se Professor. Também foi co-fundador e Professor-Pesquisador do Instituto de Estudos Internacionais (1967-1973), Coordenador do Programa de Desenvolvimento Sustentável (1994 - 2001), Diretor do Centro de Análise de Políticas Públicas (1997-2001) e Fundador e Diretor do Instituto de Assuntos Públicos (2001 - 2003).

1 Doutor em ciencia politica pela UFPE, pesquisador do Núcleo Desenvolvimento \& Região do Departamento de Pós-Graduaçao em Ciencia Politica da UFPEe pesquisador bolsista da FACEPE (Fundaçaõ de Amparo a ciencia e Tecnologia do governo do estado de Pernabuco). 
Na Development Research Corporation presidiu entre 1990 e 1994 o FÓRUM 9o, um programa conjunto do CIEPLAN, CPU, Instituto de Ciência Política da Universidade Católica e FLACSO sobre Transformação Democrática do Estado e da Sociedade Civil; e desde 1997 é Responsável, com o Presidente do Diálogo Interamericano, do Programa de Promoção da Reforma Educacional na América Latina (PREAL). Em 1975 foi nomeado Professor do Instituto de Estudos de Desenvolvimento da Universidade de Sussex (1975 - 1986). Ele também foi professor visitante da FLACSO e da Universidade Católica do Chile em Santiago, o Colégio do México, a Universidade de Paris, o Max Planck Gesellschaft, e as Universidades do Texas em Austin (Tinker Chair), Duke, Califórnia (Los Angeles) e Flórida (Bacardi Eminent Scholar Chair).

De 1969 a 1975 foi membro do Comitê Conjunto de Estudos Latinoamericanos do Social Science Research Council e do American Council of Learned Societies of the United States. Em 1992 foi eleito Membro Número da Academia de Ciências Sociais do Instituto do Chile. Em 1994 recebeu o Prêmio Kalman Silvert, a maior distinção da Associação LatinoAmericana de Estudos. Em 1995 foi nomeado Membro Correspondente da Academia Colombiana de Ciências Econômicas; em 1996 foi eleito membro do Conselho Consultivo Científico Internacional da UNESCO. Durante 1997-1998 foi Presidente do Comitê Organizador da V Conferência Bienal da Sociedade Internacional de Economia Ecológica.

É autor e coautor de mais de 30 livros e cerca de 150 artigos publicados em diversos países e idiomas sobre inflação, desenvolvimento econômico, história, socioeconomia, relações internacionais, integração latioamericana, meio ambiente e desenvolvimento sustentável.

Seu livro mais conhecido é O Subdesenvolvimento latino-americano e a Teoria do Desenvolvimento, com Pedro Paz, Século XX, México, publicado originalmente em 1970, com mais de 30 edições. ${ }^{2}$

Osvaldo Sunkel, ingressou na Cepal no ínicio dos anos 1950, após terminar o curso de formação cepalina, foi para Europa como bolsista das Nações Unidas. Retornou dois anos depois e assumiu a formação da Cepal, substituindo Jorge Ahumada, que saía em missão para Colômbia.

Anos depois, a convite de Celso Furtado, junta-se ao grupo de economistas, eles dois mais Juan Noyola e Óscar Soberón, para elaborar um estudo sobre a economia mexicana, ali permanecendo durante quatro anos.

2 Fonte: Cepal https://www.cepal.org/es/equipo/osvaldo-sunkel 
De volta a Santiago, foi indicado para gerir o escritório da Cepal no Rio de Janeiro. Era o ano de 1958 e Celso Furtado voltava de Cambridge, com o livro Formação Econômica do Brasil recém publicado, e assumia uma diretoria no Banco de Desenvolvimento Econômico, ainda sem o social, renunciando a seu cargo nas Nações Unidas. Sunkel viu o movimento que "Uma política de desenvolvimento para o Nordeste", a Operação Nordeste, o Codeno e, meses depois, a Sudene provocaram no Brasil. Organizou o primeiro curso de formação de profissionais do desenvolvimento, condição primeira para fazer parte dos quadros daquela nova instituição e, para tanto, viajou o Nordeste, de avião, de carro, de ônibus, e viu "físicamente" a pobreza e a miséria.

Como Celso Furtado, pôs em prática a teoria da relação centro-periferia. Acredita na atualidade da obra furtadiana e a considera absolutamente indispensável para qualquer pessoa que queira compreender a história das últimas décadas.

\section{Entrevista}

- Como era su visión de America Latina? Y del desenvolvimiento para la región? Nos gustaría saber, para tener conocimiento de las ideas de Celso Furtado, como fue su llegada. Lo dejo para que se explaye.

Bueno, yo en realidad conocí a Celso Furtado cuando él ya estaba en la Cepal. Él lleva varios años allís, cuando yo llegué a la Cepal en el año 1951, o 1952 como estudiante. La Cepal se creó en el año 1948, se formó con un grupo de jóvenes economistas latinoamericanos, entre los cuales trajo a Celso Furtado, con Noyola de México, con Regino Boti $^{5}$ de Cuba, con Jorge Ahumada de Chile. En fin, un grupo muy importante de gente bien formada en esa época en que generalmente venían de universidades extranjeras, y con muy buena formación. El

3 Celso Furtado foi para a Comissão Econômica para América Latina e Caribe - Cepal em 1949, convidado pelo mexicano Gustavo Martínez Cabañas (1911 -2003), primeiro secretário executivo (1949-1950), permanecendo por nove anos.

4 Juan Noyola Vasquez (1922 -1962), trabalhou na Cepal entre 1950 e 1959 e chefiou a missão Cepal em Cuba 1959-196o, ao término, permaneceu no país e criou a Junta Central de Planificação, permanecendo até sua morte.

5 Regino Boti (1923 -1999) Ministro da Economia de Cuba (1959-1960). 
chileno Jorge Ahumada ${ }^{6}$ que creó un curso de la Cepal, el primero de esos cursos se realizó no recuerdo bien si en el año 1950, o 1951 y a ese primer curso fue al que llegué yo. Yo llegué a la Cepal como estudiante, un estudiante que todavía no terminaba mi formación universitaria, mi carrera en la Universidad de Chile. Llegué como estudiante y estuve un año prácticamente en un curso que daba Jorge Ahumada, lo daba él solo, era básicamente un curso de lecturas y discusión, fundamentalmente, y con algunas exposiciones, con algunos de los economistas más destacados de la Cepal, por supuesto. Mi primera impresión de Celso fue el de un expositor, uno de los miembros más importantes de la Cepal, que hacía unas clases en el curso. Yo después terminé ese curso, y me dieron una beca, para estudiar en el extranjero, una beca de Naciones Unidas, que se daba al mejor estudiante del grupo. Era un grupo pequeño éramos 11012.

\section{- Era pequeño, pero los mejores...}

Era cada uno muy extraordinario, y todos de diferentes países. Después terminé ese curso, me fui a Inglaterra, donde estuve dos años, y además viajando por varias universidades europeas, visitando Europa, visitando varios lugares. Hago esta introducción porque recién a mi regreso, yo estaba en la disyuntiva después de dos años de hacer un magister pensaba en el doctorado, cuando recibí una carta de Jorge Ahumada. Me decía, "yo me voy a ir a Colombia, a una misión y quiero que vuelvas para hacerte cargo de los cursos". Yo tuve que decidir si hacia mis estudios superiores, y decidí volver a la Cepal porque en aquella época, comienzos de la década de los 1950, las investigaciones sobre el desarrollo económico eran lamentablemente algo casi desconocido, solamente había un seminario sobre lo que se estaba publicando en Latinoamérica, no se había oído hablar de la Cepal por supuesto, entonces yo regresé y me hice cargo del curso que daba la Cepal.

\section{- Profesor, cual su vinculo con él (Celso Furtado)?}

Yo me hice digamos, compañero, amigo, alumno, discípulo de Celso en esa época, en el año 1956. Celso fue destinado a México, para organizar un grupo y hacer un estudio de la economía mexicana, y ahí

6 Jorge Ahumada Corvalán (1917 - 1965) foi um destacado economista chileno, trabalhou na Cepal entre 1950 e 1960. 
me llamó, para que fuera a trabajar con él, en ese grupo. Él estuvo 4 años, y ahí después de hacer el estudio de México. El informe sobre México era un informe técnico, que no le gustó al gobierno mexicano. Porque ya Celso defendía en aquella época que la crisis del desequilibrio estructural del sector externo de la economía latinoamericana, que tendía sistemáticamente a un déficit en la balanza de pago con exceso de importaciones y déficit de exportaciones, y que entonces las devaluaciones que ocurrían en México daban en una deficiencia estructural económica, y eso el gobierno mexicano rechazó ese estudio. Ese informo produce en conflicto entre la Cepal y el gobierno mexicano: Furtado quería que ese estudio se publicara en la Cepal, pero el gobierno mexicano no se lo permitió. Y entonces, ahí Furtado renunció $^{7}$ a Cepal. Yo me quedé en la Cepal. A esas ya nos habíamos hecho muy amigos, colaboré mucho con él en su estudio, allá en México, jugábamos tenis juntos, era un buen jugador de tenis, él después estuvo en Europa, un año en Cambridge, en Inglaterra.

\section{- Estuvo en Inglaterra y después profesor?}

Después, Celso regresó a Brasil y a mí, después de volver de México a Santiago, me designaron jefe de la oficina de Cepal en Río, donde colaboré mucho con Celso en la etapa digamos formativa de la Sudene. Estoy hablando de Furtado en la Cepal a fines de los años 1950, hasta 1959 más o menos. Después de Cambridge él regreso a Brasil, Roberto Campos organizó su entrada al Banco de Desarrollo Económico, y Furtado trabajó durante un año ahí, y produjo este primer libro, el informe sobre el Nordeste y las relaciones entre el Norte y el Sur de Brasil, la relación digamos centro-periferia brasileña y fue un informe que tuvo un enorme impacto, por el cual se creó la Sudene.

- Sabemos profesor, que usted estaba encargado de la oficina de Brasil de la Cepal, conoce muy bien usted la temática del Nordeste?

Bueno, esa primera temática, tuvo resultado cuando estaba trabajando en Cepal sobre el tema de las estratégicas de proyecciones y

7 Na realidade, Celso Furtado licenciou-se da Cepal, e a convite do economista inglês Nicholas Kaldor e com uma bolsa da Fundação Rockefeller, estudou em Cambridge, em 1958, quando escreveu Formação Econômica do Brasil. In Diários Intermitentes, Rosa Freire D’Aguiar, Companhia das Letras, 2019 e Obra autobuiográfica, Celso Furtado, Companhia da Letras, 2014. 
de planificación económica, hubo una serie de estudios que estaba haciendo en varios países latinoamericanos, sobre proyecciones, análisis del pasado, presente, diagnósticos, planificaciones y proyecciones de análisis económico para el futuro de nuestros países, entre los cuales también se hizo uno para Brasil. En realidad, son recuerdos que digamos me vienen en la memoria (medida) ahora que estoy conversando con usted. Recuerdo dos o tres motivos por los que se generó un interés especial con Celso. Uno fue un motivo político nacional: en el Nordeste comenzaba a haber influencias de grupos revolucionarios, y eso fue, en circunstancias en que acababa de ocurrir una revolución cubana, el Che Guevara andaba en Bolivia, y había en Brasil mucha preocupación sobre el tema de la pobreza y la posibilidad de que pudiera haber movimientos revolucionarios. Hubo una gran influencia norteamericana que después también se manifestó en la Alianza para el Progreso. La segunda yo creo que fue directamente el interés, una cosa más general. Yo creo que en aquella época comenzó un interés creciente por el desarrollo regional. En general en América Latina, el desarrollo regional pasó a ser un tema de significación, y lógicamente el caso brasileño, el subdesarrollo del Nordeste en contraste con el desarrollo de Sao Paulo. Pero además, yo creo que en el caso de Celso en particular, el Nordeste era el objetivo, Celso nació en Paraíba, estudio en Recife y él era nordestino además físicamente, el tipo humano de Celso era el tipo humano que se ve en el Nordeste de Brasil. Entonces, tenía su corazoncito en el Nordeste. Y había también el interés del gobierno por tratar de equilibrar el desarrollo brasileño, de todo el desarrollo fuera en la región de Sao Paulo. De ahí surge su idea de crear la capital de Brasil en Brasilia, en el interior, o sea el desarrollo del interior y en particular, del Nordeste, el famoso polígono de la seca, que era una preocupación muy grande. El interés de Celso por el Nordeste en el Banco de Desarrollo, la Operación Nordeste, se produjo entonces, el libro ${ }^{8}$, y nosotros desde el escritorio de Cepal colaboramos en esa tarea. Posteriormente cuando ya se había creado la Sudene, Celso le pidió a Cepal que organizara un curso de formación de futuros posibles funcionarios de la autarquia, y me lo encargaron a mí, porque yo estaba

8 Provavelmente o entrevistado se refere a "Uma Política para o Desenvolvimento do Nordeste", com estudos do GTDN - Grupo de Trabalho para o Desenvolvimento do Nordeste. 
a cargo de los cursos de capacitación en la Cepal de Río de Janeiro. Organizé, en Recife un curso de desarrollo económico, que duraba aproximadamente tres meses, tenía una serie de componentes, un curso introductorio sobre desarrollo económico, con cuestiones muy básicas, cuentas nacionales, proceso de desarrollo económico, formación de capitales, equilibrio externo, etc. El curso introductorio que yo daba, y después había una serie de otros cursos, que recuerdo ahora, un curso muy importante de formulación y evaluación de proyectos, recuerdo a un alumno de ese primer curso, Antonio Baltar, lo recuerdo mucho porque era una persona ya mayor. Bueno, yo me fui a Recife y residí allá durante mas o menos tres meses, previo al curso, porque la idea de Celso era que yo recorriera todos los estados del polígono de las secas, desde Bahía hasta el Maranhão. Me designaron un secretario que no recuerdo su nombre, Celso trajo gente de muchos lados, recorrimos físicamente todos los estados del Nordeste, que eran parte del espacio cubierto por Sudene que organizó previamente un concurso, para que postularan a personas de los distintos estados, que tuvieran méritos e intereses, en trabajar en la nueva dirección que se creaba. Ese periodo prácticamente tuvo mucho rechazo, y se hacía mucha batalla en la creación de este nuevo organismo, los que lo rechazaban, los que querían incorporarse, una batalla para tener el privilegio de ser funcionarios y una de las tácticas que diseño Celso para evitar un poco la avalancha de presiones fue realizar este curso, para así poder decir estos señores son los mejores alumnos, del curso preparatorio entonces yo los voy a elegir. Y de ahí salieron después con la experiencia del curso hace años salieron una serie de expertos brasileños cuyos nombres no recuerdo ya, pero que de vez en cuando me los encuentro cuando voy a Brasil.

Entonces, yo fui por todos los estados recorriendo durante dos o tres semanas, entrevistando personas, entrevistamos 150 personas, y fuimos entrevistando y en la medida en que fuimos entrevistando fuimos seleccionando dos o tres de cada estado como candidatos para el curso. El curso se realizo a fines, y esto debería verificarlo, a fines del año 59, yo recuerdo haber celebrado mis 30 años en Recife durante el curso. Y bueno, realizamos el curso con este grupo, resultó ser un grupo muy grande, eran como 60 alumnos, fue muy interesante, recoger las experiencias de lo que decía la gente de los distintos estados, y posteriormente Celso, bueno, hicimos una evaluación final del curso, de los alumnos que participaron en ese curso, por lo menos unos 20 
quedaron efectivamente, fueron convocados ya para ser funcionarios de la Sudene. No me acuerdo de los nombres, pero Baltar ${ }^{9}$ era uno de ellos, era un señor ya de bastante edad, y después resulto ser un gran experto en los proyectos. Recorrí todo el norte, toda la zona, y aprendí muchísimo de lo que era la pobreza, realmente no puedo olvidarme, íbamos en el avión de una capital a la otra, no era fácil porque no había conexiones muy frecuentes, y precisamente para llegar a Piauí, había solo un avión semanal, que volaba de Fortaleza a Piauí, pero era un avión semanal y en Piauí después de dos días no había absolutamente nada que hacer, no había nadie mas que entrevistar, era pobrísimo, de manera que nos volvimos en autobús, de Piauí a Fortaleza, y ese fue yo diría mi primer encuentro con la miseria, la pobreza extrema, y ahí en el autobús íbamos 30 pasajeros, 20 por lo menos era gente que emigraba, que salía de Piauí para irse a Fortaleza y después al sur, Eldorado para ellos. Y una de las cosas que no voy a olvidar jamás, la pobreza se manifestaba físicamente, cuando el bus llegaba a algún lugar y paraba, creaba lo que uno ve en las películas de la India, de África, ver a los niños y a los viejos ciegos, deformaciones físicas, demacrados, la pobreza física, la expresión física de la pobreza y de la miseria. Y recuerdo que al mediodía el bus paraba en algunos de los pueblos entre Fortaleza y Piauí, y ahí se daba el almuerzo, en unas tiendas que eran como restaurante, y el almuerzo era un plato de arroz con huevo frito, yo observé que mucha de la gente que estaba ahí no se comía el huevo, me pareció extrañísimo, le pregunté a mi secretario, el dijo que no conoce no sabe lo que implica, obviamente no tenían gallinero, no había una cosa de ese tipo, en sus lugares de origen, a mi me impresionó pero era efectivo que los huevos quedaron ahí, no se los comieron.

- Profesor cual sería la vigencia de Celso sobre todo hoy, en su pensamiento en el desarrollo latinoamericano, posicionado y globalizado, cual sería su pensamiento y también el pensamiento de los economistas, de aquella época, si lo convirtiéramos a un momento actual. Cuales serian las ideas

9 Antônio Bezerra Baltar (1915-2003) Engenheiro, professor, urbanista e político, foi suplente de senador, foi aposentado pelo Ato Institucional Número Um. De 1965 a 1977 foi para Santiago do Chile a convite da Cepal, em 1990 foi eleito professor emérito da Universidade Federal de Pernambuco. 
principales que serian de importancia para Celso Furtado, para los cepalinos, parecería ser que la relación que la Cepal tiene que trabajaba con tanta intensidad por el desarrollo regional continuar haciendo una de sus gestiones.

Sin duda, en primer lugar la obra de Celso es una obra definitiva clásica, del pensamiento sobre el desarrollo en general, del mundo, del pensamiento sobre el desarrollo de América Latina, en ese lugar Celso Furtado es una figura de la misma estatura de Prebisch ${ }^{10}$ que Urquidi ${ }^{11}$ en México, de los grandes pensadores de América Latina, tiene su pensamiento calificando la formación y la relación del país con el mundo, va teniendo con los holandeses, con el África, con Inglaterra, con Francia, con EE UU, como se va planteando a lo largo de la distinta configuración del mundo, y va de alguna manera como reproduciendo como una especie de espiral, donde el gran enfoque centro periferia sigue siempre estando en el eje del pensamiento. Y eso vinculado con la estructura social, económica y política interna, e incluso la dimensión cultural que él agregó en los últimos libros que él escribió: en realidad, el desarrollo es un proyecto cultural, y también no ha logrado formar todavía, un el dialogo (entre el desarrollo económico y como proyecto cultural). Yo creo que el pensamiento de Celso sigue absolutamente vigente, es una lectura obligatoria, para cualquiera que esté interesado en desarrollo hoy en día, y ciertamente me gustaría muchísimo preguntar todo esto que está sucediendo en los últimos 20 años o algo así, que se está reconfigurando completamente el mundo, con las grandes transformaciones geoeconómicas, políticas, demográficas, la incorporación de media humanidad, a la modernidad en China y Asia, el balanceo de los ejes de poder y la economía mundial hacia Asia y el proceso de Europa y EE UU, todo eso está digamos, si uno piensa como Celso, estas cosas se hacen perfectamente bien hoy en día, y sigue estando presente esta necesidad de tener pensamiento propio, esa es otra cosa en que Celso insistió muchísimo, la posibilidad

10 Raul Prebisch (1901 - 1986) foi secretário executivo da Cepal de 1949 a 1963 quando deixou a instituição a pedido do Secretário Geral da ONU, U Thant, para assumir o cargo de Secretário Geral da UNCTAD (Conferência das Nações Unidas sobre Comércio e Desenvolvimento onde ficou até 1969.

11 Victor Luis Urquidi Bingham (1919-2004) Economista mexicano, 1941 ingressou no Departamento de Estudos Econômicos do Banco de México. Trabalhou também no Ministério das Finanças e Crédito Público e na Comissão Econômica para a América Latina e o Caribe (CEPAL). 
de un pensamiento propio, que los países de América Latina tengan una cultura de desarrollo que venga desde dentro. Hay un libro mío por ahíi ${ }^{12}$, el desarrollo desde fuera o desde dentro, y me parece que también es una forma de poner la idea de un foco propio autónomo, autentico, racional, sociocultural y político, para ahí realmente hacer un proceso de desar-

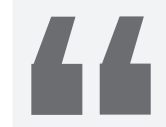

De manera que yo pienso que el pensamiento de Celso es absolutamente vigente, $\mathrm{y}$ absolutamente indispensable para cualquier persona que quiera entender todo lo que ha estado pasando en las últimas décadas.

rollo moderno. De manera que yo pienso que el pensamiento de Celso es absolutamente vigente, y absolutamente indispensable para cualquier persona que quiera entender todo lo que ha estado pasando en las últimas décadas. Esa fue un poco mi cercanía con Celso, y después durante un largo tiempo casi a unos pocos años de su muerte, nos encontrábamos con alguna frecuencia en conferencias, en visitas que él hacia o que yo hacía a Brasil, en reuniones después en Paris mucho tiempo después, se desarrolló una amistad muy grande con él. Lo respeto por su capacidad, por su trabajo, su personalidad, verdaderamente potente, un pensador prácticamente con una gran trayectoria.

12 El desarrollo desde dentro: un enfoque neoestructuralista para la América Latina, Ciudad de México, Fondo de Cultura Económica, 1991. Osvaldo Sunkel. 